\title{
APRENDIZAGEM E DECISÃO ESTRATÉGICA REALIZADAS POR EQUIPES DE DIREÇÃO EM PEQUENAS EMPRESAS
}

DOI: 1014211/regepe.v5i3.383

Artigo recebido em:22/06/2016

Artigo aprovado em: 28/10/2016

Tatiane Silva Tavares Maia - Universidade Federal do Mato Grosso do Sul - UFMS ${ }^{1}$ Edmilson Lima - Universidade Nove de Julho - UNINOVE ${ }^{2}$

Resumo: Este artigo tem por objetivo gerar conhecimentos sobre como e por que ocorre a aprendizagem em apoio ao processo de decisão estratégica em equipes de direção de pequenas empresas (PE). Para tanto, o processo foi estudado detalhadamente em quatro PEs. A abordagem metodológica é descritiva, baseada em métodos qualitativos e no estudo multicaso. Os dados foram coletados principalmente com entrevistas em profundidade e gradativamente analisados com procedimentos de análise intra e intercaso, conforme as recomendações de Miles e Huberman (1994). Os resultados mostraram que o comportamento dos codirigentes no processo de decisão estratégica combina mais com a aprendizagem do que com procedimentos de análise de projeções numéricas ou de cenários futuros, por exemplo. A descrição sobre como os processos de decisão estratégica ocorreram nas PEs é bem diferente dos modelos identificados na literatura. Uma característica de destaque é que se mostraram bem simples, mais baseados em diálogos ocorridos nas equipes de direção, até mesmo informais, do que em análises racionais muito elaboradas.

Palavras-chave: Aprendizagem em Circuito Duplo; Processo de Decisão Estratégica; Equipe de Direção; Pequena Empresa.

\section{LEARNING AND STRATEGIC DECISION BY MANAGEMENT TEAMS IN SMALL BUSINESSES}

\begin{abstract}
This article aims to generate knowledge about how and why learning occurs in support of strategic decision-making in small business management teams (PE). Therefore, the process has been studied in detail in four PEs. The methodological approach is descriptive, based on qualitative methods and multi-case study. Data collection was done mainly through in-depth interviews, whose analysis included two stages, in-case analysis and inter-case as the Miles and Huberman
\end{abstract}

\footnotetext{
${ }^{1}$ Endereço: Av. Pedro Pedrossian, 725, Bairro Universitário, Paranaíba - MS, CEP. 79500-000.

E-mail: tstavares@gmail.com

2 E-mail: edmilsonolima@gmail.com
}

TAVARES, T. S.; LIMA, E. Aprendizagem e decisão estratégica realizadas por equipes de direção em pequenas empresas. Revista de Empreendedorismo e Gestão de Pequenas Empresas, v.5, n.3, 2016. 
(1994) recommendations. The survey results showed many ideas about how learning contributes to the process of strategic decision on PEs. It was found that the behavior of these processes together combines more with learning double circuit than rational analysis procedures. A description of how the strategic decision processes occurred in that PEs is quite different from ready models in the literature. A prominent feature is that it showed very simple, more based on dialogues occurred in management teams, even informal, than very elaborate rationales.

Keywords: Learning in Double Loop; Strategic Decision Process; Management Team; Small Business.

\section{Introdução}

A literatura sobre o processo de decisão estratégica na pequena empresa (PE) é escassa (ALCANTAR; NGWENYAMA, 2015). Portanto, escassos também são os estudos empíricos que descrevem como os dirigentes dessas organizações tomam suas decisões estratégicas e quais elementos influem nesse processo colaborando para eficácia deste último.

A decisão estratégica é entendida aqui como o conjunto das escolhas intencionais ou respostas programadas sobre questões que afetam significativamente a saúde e a sobrevivência da organização (EISENHARDT; ZBARACKI, 1992). Decisões de desenvolvimento de um novo produto, entrada em um novo mercado, reestruturação da organização ou posicionamento de forma diferente no mercado, em vez de simples tratamento de questões de rotina, são exemplos de decisão estratégica, como destacam Liberman-Yaconi, Hooper e Hutchings (2010).

Agregar novos insights sobre os processos de decisão estratégica na PE representa uma contribuição para a literatura investigativa sobre a gestão estratégica desse tipo de empresa, tão carente de desenvolvimento e aprofundamento quando comparada à vasta literatura sobre a decisão estratégia em grandes empresas (BROUTHERS; ANDRIESSEN; NICOLAES, 1998; ALCANTAR; NGWENYAMA, 2015). Dada a carência de pesquisa acerca da decisão estratégica na PE e a importância do tema, este trabalho busca contribuir na compreensão do processo, particularmente quanto ao realizado por uma equipe de direção.

TAVARES, T. S.; LIMA, E. Aprendizagem e decisão estratégica realizadas por equipes de direção em pequenas empresas. Revista de Empreendedorismo e Gestão de Pequenas Empresas, v.5, n.3, 2016. 
As PEs comumente enfrentam dificuldades associadas a sua fragilidade provocada principalmente pela acentuada restrição de recursos, não só financeiros, e sua suscetibilidade às mudanças e ao dinamismo do contexto dos negócios. São desafios típicos de sua realidade, frente aos quais a condução da empresa por uma equipe de direção ajuda a somar competências, ampliar a capacidade decisória, empregar múltiplas redes de relação dos codirigentes (membros de uma equipe de direção) a favor do negócio e explorar mais recursos, inclusive financeiros, do que ofereceria apenas um dirigente.

Embora haja o reconhecimento de que a equipe de direção desempenha um papel significativo em qualquer organização (HAMBRICK; MASON, 1984), no caso da PE, ela é particularmente importante por facilitar o acesso a capacidades e recursos principalmente pela complementaridade entre codirigentes e a rede de relação oferecida por cada um deles (GREENE; BRUSH; BROWN, 2015; LOHRKE; FRANKLIN; KOTHARI, 2015). O processo de decisão estratégica, central para o desenvolvimento das organizações em geral, mas sobremaneira marcado por riscos redobrados nas PEs por elas serem frágeis, fortalece-se com uma equipe de direção, em especial por conta das interações construtivas e da aprendizagem coletiva dos codirigentes (LIMA, 2010, 2012; BRESMAN; ZELLMER-BRUHN, 2013; YOON; KIM; SONG, 2015; WOLFF; PETT; RING, 2015).

Para esse tipo de empresa, novos estudos justificam-se diante da necessidade de modelos conceituais particularizados visando mais conhecimento sobre o processo de decisão estratégica, dado que os estudos do tema raramente focam a PE (COOPER, 1981; FAGUNDES; GIMENEZ, 2009). Dada sua fragilidade, as PEs enfrentam não apenas mudanças que chegam a abalar inclusive grandes empresas, mas também aquelas que atingem as PEs sem impor problemas às empresas maiores.

Nesse sentido, as PEs são forçadas a ajustes mais frequentes, cujas razões podem incluir mudanças contextuais relacionadas à legislação, ao desenvolvimento e à aplicação de novas tecnologias, assim como a ações dos concorrentes. É comum que tais mudanças imponham ajustes incoerentes com práticas e pensamentos precedentes nas empresas (BROUTHERS; ANDRIESSEN; NICOLAES, 1998), tornando essencial a aprendizagem das equipes de direção em direção em pequenas empresas. Revista de Empreendedorismo e Gestão de Pequenas Empresas, v.5, n.3, 2016. 
apoio a sua tomada de decisão estratégica (CARMELI; TISHLER; EDMONDSON, 2012; GELDENHUYS; CILLIERS, 2012).

Para o avanço dos estudos sobre tomada de decisão estratégica em equipes de direção, além do habitual enfoque em aspectos demográficos, é necessário o enfoque dos processos coletivos que permitam entender como e porque tal tomada de decisão ocorre nas equipes (OLSON; PARAYITAM; BAO, 2007; LIU; MAITLIS, 2014). Um desses processos é a aprendizagem coletiva dos codirigentes (CARMELI; TISHLER; EDMONDSON, 2012; GELDENHUYS; CILLIERS, 2012; LIMA, 2012; BRESMAN; ZELLMER-BRUHN, 2013; YOON; KIM; SONG, 2015; WOLFF; PETT; RING, 2015).

Quando se trata de como e o porquê da tomada de decisão e da aprendizagem em equipes de direção de PE, a carência de novas pesquisas é ainda maior, segundo o que o texto já sinaliza acima. Nesse sentido, o objetivo do presente trabalho é gerar conhecimentos sobre esses dois processos em equipes de direção em PE.

O artigo está estruturado em seções. A primeira traz o referencial teórico quanto às equipes de direção na $P E$, à aprendizagem organizacional e aos processos de tomada de decisão estratégica em PE. Em seguida, apresentam-se os métodos do estudo. Três outras partes complementam o trabalho, contendo a apresentação e a análise, respectivamente, dos casos de PE estudados, dos processos de decisão estratégica identificados nelas e da aprendizagem associada a tais processos. Uma seção de considerações finais encerra este trabalho.

\section{Equipes de Direção e PE}

A equipe de direção de uma PE é entendida aqui como a equipe formada por coproprietários-dirigentes atuantes na decisão estratégica: os codirigentes. Segundo Hambrick (1995), uma equipe pode ser definida como uma coletividade composta de pessoas unidas e interdependentes com a finalidade de realizar atividades em conjunto. Seus membros normalmente têm diferenças de habilidades e capacidades, o que permite maior cobertura de diferentes áreas de atividade da 
empresa na comparação com a atuação de uma única pessoa, havendo tendência à complementaridade (EISENHARDT; KAHWAJY; BOURGEOIS III, 1997; LIMA, 2012; LIU; MAITLIS, 2014; YOON; KIM; SONG, 2015).

Considerando-se o trabalho de Finkelstein e Hambrick (1996), essa definição mostra-se condizente com os estudos da PE, pois, nessas organizações, os dirigentes normalmente são também proprietários da empresa, o que lhes confere grande poder de participação na tomada de decisão. Uma particularidade fundamental da PE respeitada pela definição é a preponderância dos proprietáriosdirigentes nesse tipo de organização. Nas PEs, os dirigentes estão no centro das atividades, cabendo a eles exercer um importante papel na definição das ações estratégicas. Com uma equipe de direção, esse papel é compartilhado e acredita-se que as chances de sucesso da PE aumentam (LECHLER, 2001; RUEF; ALDRICH; CARTER, 2003; LIU; MAITLIS, 2014).

Comparadas à situação das PEs administradas por apenas um proprietáriodirigente, aquelas administradas por equipes apresentam como vantagens: maior capacidade de investimento, mais diversidade, maior base de conhecimento, relações e experiências a serem utilizadas em benefício do desenvolvimento dos negócios (LIMA, 2012; LOHRKE; FRANKLIN; KOTHARI, 2015). Por esses motivos, é comum os dirigentes juntarem-se em equipes, tornando seus negócios frutos não apenas de iniciativas individuais (PECH-VARGUEZ et al., 2010).

Ao se estudarem equipes de direção de PE, é possível encontrar características específicas que as diferenciam das equipes de direção de grandes organizações. As equipes de direção de PE estão continuamente considerando as restrições típicas de recursos que essas empresas precisam superar, relacionadas com as vendas e o marketing, o planejamento, a falta de experiência administrativa dos dirigentes e as restrições de recursos financeiros (WU; YOUNG, 2002; FULLERLOVE, 2006). As atitudes e habilidades da equipe de direção são determinantes dos resultados das PEs que administram (BRUNNINGE; NORDQVIST; WIKLUND, 2007; ESCRIBÁ-ESTEVE; SANCHEZ-PEINADO; SANCHEZ-PEINADO, 2009).

Nessas empresas, não se espera encontrar uma estrutura organizacional complexa e procedimentos administrativos sofisticados (ESCRIBÁ-ESTEVE; SANCHEZ-PEINADO; SANCHEZ-PEINADO, 2009). Geralmente não há recursos direção em pequenas empresas. Revista de Empreendedorismo e Gestão de Pequenas Empresas, v.5, n.3, 2016. 
com folga ou sistemas elaborados de gestão, como em grandes empresas, sustentando os processos decisórios. Consequentemente, as PEs contam mais com as habilidades de seus proprietários-dirigentes (REUBER; FISCHER, 1997; LUBATKIN et al., 2006) do que com o uso de técnicas refinadas e/ou de procedimentos racionais-analíticos detalhados.

Liberman-Yaconi, Hooper e Hutchings (2010) estudaram a tomada de decisão estratégica em microempresas, descrevendo tal decisão também na realidade das PEs. Os autores destacam que nessas empresas as decisões ocorrem normalmente dentro dos limites do mundo cognitivo dos dirigentes, sendo a tomada de decisão estratégica fortemente influenciada por suas experiências, valores e habilidades de gestão (LIBERMAN-YACONI; HOOPER; HUTCHINGS, 2010). Por isso é importante observar a composição e as demais características das equipes de direção e de seus membros para se entenderem as decisões estratégicas realizadas por elas.

Adicionalmente, as relações de colaboração e cooperação, a tomada de decisão conjunta, a troca de informações, o compartilhamento de habilidades também são aspectos importantes para o funcionamento da administração feita por equipes de direção (LUBATKIN et al., 2006). Para Tihula e Huovinen (2009), as melhores equipes de direção são aquelas que entendem e exploram essa realidade, além de desenvolver a capacidade de resolver grandes problemas e tomar decisões rapidamente com o empenho de todos seus membros. Segundo os autores, o valor das equipes de direção reside na capacidade de mobilizar esforços coordenados dos codirigentes.

O trabalho em equipe de direção possibilita aos dirigentes desenvolver um processo coletivo e participativo por meio do qual os membros da equipe possam interagir e lidar com questões difíceis, tomar decisões estratégicas importantes e construir compromisso (CARMELI, 2008; CARMELI; TISHLER; EDMONDSON, 2012). Nestas equipes, os codirigentes se reúnem para compartilhar informações, perspectivas e conhecimentos e, diante disso, tomar decisões com embasamento.

Pode-se perceber que diferentes fatores relacionados à composição da equipe têm impacto direto nessas decisões, tais como: heterogeneidade funcional, diversidade cognitiva, idade e experiência da equipe. Uma compreensão mais direção em pequenas empresas. Revista de Empreendedorismo e Gestão de Pequenas Empresas, v.5, n.3, 2016. 
completa sobre os fatores ajuda a entender os processos de tomada de decisão (ESCRIBÁ-ESTEVE; SANCHEZ-PEINADO; SANCHEZ-PEINADO， 2009). A empresa como um todo é beneficiada quando os codirigentes têm alta capacidade cognitiva, traços de personalidade propícios a boas relações e competências favoráveis à complementaridade e à cobertura das atividades necessárias na empresa.

\section{Aprendizagem na Gestão Estratégica}

Argyris e Schön (1978) explicam o processo de aprendizagem por meio de dois circuitos diferentes. O primeiro é denominado circuito único (single loop). Ele envolve um ajuste às práticas existentes, com foco na obtenção de melhorias nos processos e sistemas de trabalho. Volta-se para as atividades cotidianas e para o "como fazer". O segundo é o circuito duplo (double loop) e inclui, além dos componentes do circuito único, o questionamento do quadro mental vigente que orienta a ação, o que possibilita a criação de novos conhecimentos e a reflexão sobre possíveis inconsistências (ARGYRIS, 1976).

Nesse caso, a aprendizagem não se restringe apenas a questões operacionais e inclui a revisão dos porquês das ações, dos modos de se administrar e das estratégias. Como reforçam Kantamara e Vathanophas (2014), esse tipo de aprendizagem ocorre com o questionamento e a modificação de normas subjacentes, procedimentos, políticas e objetivos.

A distinção crucial entre os circuitos relaciona-se, portanto, com 0 aprofundamento do processo de aprendizagem organizacional. O processo de aprendizagem em circuito duplo se constitui numa espécie de questionamento organizacional que interroga os valores que guiam as estratégias de ação, altera ou elimina as normas organizacionais incompatíveis com o funcionamento eficaz das empresas (EASTERBY-SMITH et al., 2004).

Pode-se dizer que o circuito duplo define novas prioridades ou reestrutura as existentes, reformulando a lógica que guia o desenvolvimento da organização. $\mathrm{Na}$ aprendizagem em circuito duplo, aprender significa refletir criticamente sobre o 
próprio comportamento e identificar as formas que, muitas vezes, inadvertidamente, contribuem para os problemas da organização, para então modificar a forma de agir (ARGYRIS, 2002). Com base na aprendizagem em circuito duplo, a aprendizagem organizacional é um processo de mudança de pensamento e ação, individual e compartilhado, reorientando os processos estratégicos (ARGYRIS; SCHÖN, 1978).

Diferentemente do que se vê quanto às grandes organizações, são raros e ainda muito necessários os estudos sobre a aprendizagem em circuito duplo em PE (CHASTON; BADGER; SADLER-SMITH, 2001; WOLFF; PETT; RING, 2015). Um desses raros estudos é o de Sapie et al. (2015). Eles focaram a aprendizagem em circuito duplo no desenvolvimento de capital humano das PEs. Outro desses estudos é o de Smith e Barrett (2014), que trata do uso de um fórum informal de discussão on-line para incentivar, apoiar e promover a aprendizagem em circuito duplo de proprietários-dirigentes de PE.

\section{Decisão Estratégica e Aprendizagem nas PEs}

Muitos estudos sobre gestão estratégica já foram feitos acerca das PEs segundo diferentes perspectivas. Dentre estudos descritivos e normativos do tema, predominam os primeiros. Com isto, é possível notar a necessidade de estudos descritivos em estratégia de PE ainda nos tempos atuais (MCCARTHY; LEAVY, 2000; WYER; MASON; THEODORAKOPOULOS, 2000; MAZZAROL; REBOUD, 2009; LIMA, 2010; MUELLER; NAFFZIGER, 2015; IBRAHIM; DUMAS; MCGUIRE, 2015).

A condução de processos de decisão estratégica à maneira da perspectiva tradicional como um processo periódico e formal não é suficiente para lidar com a realidade de gestão da PE. Nesse tipo de organização, os processos de decisão estratégica tendem a assumir formas mais plásticas (VERREYNNE; MEYER; LIESCH, 2014), as quais condizem com a realidade típica de restrição de recursos (ALEXANDERSSON; FRIDOLF, 2015) e as suas dificuldades de previsibilidade (FULLER-LOVE, 2006).

TAVARES, T. S.; LIMA, E. Aprendizagem e decisão estratégica realizadas por equipes de direção em pequenas empresas. Revista de Empreendedorismo e Gestão de Pequenas Empresas, v.5, n.3, 2016. 
As PEs não requerem alto nível de formalização, devido à habilidade do proprietário-dirigente em controlar e rapidamente comunicar as informações via mecanismos informais (MAZZAROL; REBOUD, 2009). Verreynne, Meyer e Liesch (2014) defendem, com base nos resultados de pesquisa empírica junto a 320 PEs, que é comum encontrar processos informais de decisão estratégica. Além disso, no estudo conduzido por Alexandersson e Fridolf (2015), dá-se destaque à realidade peculiar experimentada pelas PEs: uma realidade de restrição de recursos que, segundo os autores, termina por acarretar menor acesso a informações relevantes, principalmente se comparado a grandes empresas, contribuindo com a informalidade nos processos de decisão estratégica.

Liberman-Yaconi, Hooper e Hutchings (2010) defendem ainda que a tomada de decisão em empresas de porte reduzido relaciona-se mais a processos caóticos e interativos e menos à formalização, à sistematização e à linearidade. Além disso, concentra-se em poucos indivíduos, é mais centralizada, menos complexa e menos coordenada. Portanto, modelos racionais de tomada de decisão não se aplicam diretamente à realidade da PE (BROUTHERS; ANDRIESSEN; NICOLAES, 1998; LIBERMAN-YACONI; HOOPER; HUTCHINGS, 2010; VERREYNNE; MEYER; LIESCH, 2014; ALCANTAR; NGWENYAMA, 2015; MUELLER; NAFFZIGER, 2015).

Nessas empresas, a tomada de decisão estratégica é relativamente pouco elaborada quanto ao uso de técnicas administrativas e de métodos analíticos. Isso faz com que a compreensão das coisas, a capacidade de julgamento, a intuição e as aspirações pessoais dos dirigentes sejam centrais no processo decisório (LIMA, 2010; IBRAHIM, 2015).

A aprendizagem é mais frequente e importante para a decisão estratégica das PEs do que análises formais (WYER; MASON; THEODORAKOPOULOS, 2000), o que é em grande parte determinado pela fragilidade dessas organizações e pelo relativo dinamismo de seu contexto (WYER; MASON, 1998). A aprendizagem é particularmente necessária nas PEs maduras, que enfrentam desafios crescentes, pressões sociais e a aceleração de mudanças tecnológicas, geralmente tendendo a resistir à renovação estratégica (ESCRIBÁ-ESTEVE; SANCHEZ-PEINADO; SANCHEZ-PEINADO, 2009).

TAVARES, T. S.; LIMA, E. Aprendizagem e decisão estratégica realizadas por equipes de direção em pequenas empresas. Revista de Empreendedorismo e Gestão de Pequenas Empresas, v.5, n.3, 2016. 
Independentemente disso, nas PEs, em geral, é principalmente preponderante a aprendizagem dos dirigentes, pois o processo estratégico nessas organizações é muito apoiado em seu pensamento e em suas atividades (LIMA, 2010, 2012).

\section{Procedimentos Metodológicos}

A pesquisa aqui apresentada é um estudo de casos múltiplos, de natureza qualitativa e descritiva. O método qualitativo foi de fundamental importância na pesquisa porque permitiu a consideração do contexto social do fenômeno que se estudou (MORGAN; SMIRCICH, 1980; PETTIGREW, 1992), assim como a compreensão do processo (CRESWELL, 2007) de decisão estratégica dos codirigentes, baseando-se no conceito de aprendizagem.

Optou-se pelo estudo multicasos devido ao maior potencial explicativo em relação a um único objeto e por permitir análise comparativa dos dados entre casos, além de não se restringir às peculiaridades do processo de decisão estratégica de uma única equipe de direção.

Adotou-se uma perspectiva metodológica de natureza indutivo-dedutivista, combinando aspectos propostos por Eisenhardt (1989) com alguns elementos propostos por Yin (2010). Eisenhardt (1989) propõe a utilização de métodos orientados para a construção de um quadro teórico descritivo/explicativo do fenômeno estudado por meio do estudo de casos múltiplos a partir das noções de base da teoria embasada (Grounded Theory) (GLASER; STRAUSS, 1967), de modo indutivo. Já Yin (2010) preconiza um trabalho dedutivo.

A delimitação do número de casos foi orientada pela pesquisa de campo e pela teoria, levando-se em consideração que o principal interesse do estudo de múltiplos casos é conceitual (MILES; HUBERMAM, 1994). A escolha dos casos foi intencional, por meio de amostragem teórica (STRAUSS; CORBIN, 2008), em um esforço de estabelecer amostras que oferecessem novos conhecimentos pertinentes sobre os temas estudados.

TAVARES, T. S.; LIMA, E. Aprendizagem e decisão estratégica realizadas por equipes de direção em pequenas empresas. Revista de Empreendedorismo e Gestão de Pequenas Empresas, v.5, n.3, 2016. 
A amostragem teórica foi realizada, em termos práticos, em dois eventos: um caso inicial foi selecionado, denominado caso Alpha e, com base na análise dos dados referentes a essa $\mathrm{PE}$, outros casos adicionais foram sequencialmente selecionados (PANDIT, 1996).

Mais três novos casos foram selecionados segundo algumas diretrizes, que são: verificar os resultados dos casos anteriores; ampliar o escopo de resultados do estudo; obter dados para categorias conceituais específicas surgidas no decorrer da investigação; e adicionar à amostra casos que proporcionassem exemplos de tipos extremos (EISENHARDT, 1989).

Com relação à última diretriz, foi dada atenção: ao tamanho das PEs, em termos de número de funcionários; à composição da equipe de direção (familiar, não familiar ou mista); ao tipo de atividade das PEs, que eram de base tecnológica; à localização das empresas, com casos de cidades diferentes. O número de quatro casos da amostra foi estabilizado em função da indicação empírica de que a adição de mais empresas não proporcionou mais dados, contribuindo para a cobertura e o entendimento das categorias analíticas usadas na análise dos dados (GODOI; MATTOS, 2006).

No processo de montagem da amostra de pesquisa, foram seguidos critérios mínimos estabelecidos para a escolha dos casos: a) Ser uma PE, de acordo com o critérios do IBGE/SEBRAE (até 49 empregados no setor de serviços) (SEBRAE, 2011); b) Ser uma empresa dirigida por uma equipe de direção, formada por dois ou mais proprietários dirigentes; c) Ter no mínimo três anos de existência para possuir um histórico a ser explorado quanto aos temas abordados; d) As empresas deveriam ser de base tecnológica, de modo a oferecer, com o dinamismo e a necessidade contínua de aprendizagem das empresas desse setor, uma realidade com frequência relativamente alta de ocorrência da tomada de decisão em equipes de direção com base na aprendizagem.

Optou-se por estudar PE de base tecnológica por se destacarem na incorporação de conhecimento aplicado à tecnologia, principalmente, em ambientes dinâmicos e com ruptura tecnológica, como é o caso dos setores de software (TI) e farmacêutico (biotecnologia), escolhidos para serem estudados nesta pesquisa. Neles, o dinamismo do processo decisório estratégico da PE pode ser crítico para o 
seu desempenho. Importante observar que os estudos de caso foram realizados no estado de São Paulo, pela alta concentração de empresas de base tecnológica.

A coleta de dados ocorreu nos anos 2014 e 2015. As entrevistas foram realizadas na sede de cada $P E$ estudada e tiveram duração média de duas horas. Foram entrevistados dez codirigentes encarregados da tomada de decisão estratégica, além de quatro funcionários, especificamente aqueles identificados como envolvidos na decisão estratégica e que mais acompanharam e conviveram com os codirigentes.

Os dados foram coletados por meio de entrevistas em profundidade, realizadas com os codirigentes e funcionários envolvidos nos processos de decisão estratégica e por meio de análise documental. Para a adequada condução das entrevistas, foi utilizado um roteiro semiestruturado, cuja flexibilidade possibilitou a introdução de variações de acordo com cada entrevistado. Fez-se também um aprofundamento dos tópicos por meio de questões mais específicas que emergiram durante a realização das entrevistas.

As entrevistas foram gravadas e transcritas, buscando tornar os dados obtidos mais precisos, de forma a serem analisados em relação aos seus conteúdos. Foi feita uma suplementação de dados no processo de coleta por meio de análise documental. Foram utilizadas, essencialmente, informações da website disponibilizadas por todas as PEs estudadas e panfletos institucionais, em diretrizes e manuais de funcionamento de sistemas como base para essa análise e complemento dos relatos dos casos.

O processo de análise combinou a descrição para a organização dos dados e a interpretação para explicá-los em paralelo com a coleta, com a interpretação, com a construção teórica e redação do caso. Tal simultaneidade permitiu a elaboração de uma síntese de elementos principais que ajudaram a refletir na direção dos dados coletados. A análise se dividiu em análises intra e intercaso, respectivamente (MILES; HUBERMAN, 1994). A primeira envolveu a identificação de aspectos peculiares em cada um dos casos estudados e objetivou destacar conteúdos que se mostraram mais importantes para descrever e explicar o fenômeno em estudo. Essa análise foi operacionalizada atribuindo códigos às 
unidades de significação, considerando separadamente cada caso da amostra (GLASER; STRAUSS, 1967).

Em seguida, na etapa de análise intercaso, buscaram-se as regularidades por meio de um processo de comparação a partir de descrições relativas ao fenômeno estudado resultantes da análise intracaso. Portanto, a base da análise comparativa intercaso foi a feita anteriormente de cada caso separadamente. Incluiu um esforço de identificação de fatores similares e divergentes quanto a categorias de análise de interesse para cada PE (EISENHARDT, 1989).

As diferenças e similaridades identificadas contribuíram decisivamente para a formação do potencial explicativo da teorização em curso. O processo de análise foi facilitado por meio do software de pesquisa qualitativa ATLAS/TI. Por meio da utilização de dados de texto lineares como ponto de partida, entrevistas transcritas e documentos pertinentes, iniciou-se o processo de segmentação e de codificação.

\section{As PEs Estudadas: Breve Caracterização}

A empresa Alpha, com dez funcionários, localiza-se na cidade de São José do Rio Preto (SP). Foi fundada no ano de 1992, a partir do reconhecimento de uma boa oportunidade no mercado, dada a crescente necessidade de software. Os seus principais serviços são: automação comercial, gerenciamento de tecnologia da informação e desenvolvimento de softwares para gestão empresarial, pacotes prontos e personalizados.

A equipe de direção atual, composta no ano de 2010 após a dissolução da anterior que permaneceu por quatro anos, se classifica como mista, pois é composta por dois membros de uma mesma família e um membro que não é familiar. Para formar essa nova equipe, o fundador da Alpha, após considerar as experiências do passado, a formação profissional e o perfil de cada um dos eleitos, tornou sócios dois indivíduos já atuantes na empresa, quais sejam: 1) o filho de 23 anos, cuja atuação ali já ocorria desde os 14 anos de idade; 2) um funcionário muito dedicado, contratado em 2008, que atuou em diferentes setores e destacou-se na programação de sistemas. 
A somatória de informações acumuladas sobre cada novo codirigente foi importante no desenvolvimento de suas habilidades e definição do campo de atividade que ocupariam na organização. Todos os codirigentes que nela atuam estão diretamente envolvidos na gestão estratégica. As porcentagens na sociedade são: $60 \%$ do sócio fundador, $20 \%$ de seu filho e $20 \%$ do antigo funcionário. A partir da formação da equipe de direção atual, o fundador distribuiu as funções na empresa com base nas habilidades de cada codirigente. Liberado da rotina de programação, tornou-se responsável pelas finanças da empresa. Seu filho passou a cuidar do setor de suporte e gestão de pessoas, dado seu perfil mais humanista, hábil na gestão de relacionamentos com os clientes.

Quanto ao funcionário, foi nomeado pelo fundador como o principal responsável pelo setor de programação, tendo em vista sua reconhecida competência, estimulada, inclusive, por constantes atualizações. O fundador buscou reconhecer a competência central distinta dos seus novos sócios. Ao identificar a função com a qual cada codirigente demonstrava mais afinidade, conseguiu estimular a complementaridade entre os integrantes da nova equipe de direção, fato que tem fortalecido seus processos de decisão estratégica.

A empresa Beta, localizada em Ribeirão Preto, foi fundada em 2005, como um spin off para o desenvolvimento de vacinas e imunomoduladores que atuam no controle de doenças infecciosas e câncer. Recebe apoio financeiro de projetos de órgãos governamentais, como o CNPq e a CAPES. Atualmente, com nove funcionários, atua na transferência de tecnologia, proteção da propriedade intelectual, gestão de projetos de inovação e avaliação da viabilidade tecnológica. $A$ sua equipe de direção é composta por pai e filha. Classifica-se, portanto, como familiar.

Os dois codirigentes que nela atuam estão diretamente envolvidos na gestão estratégica da empresa. As porcentagens na sociedade são: $25 \%$ da filha sócia e $75 \%$ de seu pai. Seu surgimento relaciona-se com a formação profissional do codirigente pai, médico, pesquisador e professor da Universidade de São Paulo (USP) que envolveu sua filha na gestão de seus projetos de pesquisa. Nela, as funções dos codirigentes foram delimitadas desde o princípio. O codirigente pai controla a administração científica, faz os contatos, realiza networking e traz os direção em pequenas empresas. Revista de Empreendedorismo e Gestão de Pequenas Empresas, v.5, n.3, 2016. 
serviços, enquanto a codirigente filha cuida da administração dos projetos de pesquisa e da empresa, seguindo, assim, formas diferentes de trabalho.

As atividades da Beta foram iniciadas em uma incubadora de empresas, onde foram estabelecidas diferentes plataformas tecnológicas, inclusive para P\&D. De 2005 a 2011 a empresa permaneceu nela. O prazo de incubação, normalmente de três anos, já havia vencido e foi prorrogado devido a dificuldades de encontrar as instalações compatíveis. Além da finalização de seu processo de maturação, a dificuldade de fazer as adaptações às normas da ANVISA contribuiu para sua saída da incubadora. Em situação de crise, a Beta começou a buscar parceiros comerciais que pudessem sustentar sua saída da incubadora.

Contudo, apenas em 2011 surgiu o atual parceiro industrial da empresa, uma indústria nacional do setor veterinário. A Beta tornou-se seu braço de P\&D de produtos e processos biotecnológicos. Nesse espaço foi possível desenvolver melhor os serviços laboratoriais, trabalhar de acordo com as normas propostas pelos órgãos regulatórios e controlar a qualidade.

A Gama, localizada em São José do Rio Preto e com 48 funcionários, foi fundada no ano de 1986 por três amigos profissionais da área de tecnologia da informação. Surgiu no início da era da microinformática, com prestação de serviços de representação em softwares contábeis e desenvolvimento de sistemas para segmentos diversos.

Dessa sociedade, um dos sócios fundadores permanece apenas como sócio cotista. Há cinco anos se aposentou das atividades administrativas. Hoje, nela atuam dois codirigentes, mas as porcentagens são igualitárias entre os três sócios. No ano de 1989, a empresa começou a desenvolver os primeiros sistemas para os segmentos de hotelaria e CEASA, em versão DOS, ainda em sede improvisada na casa de um dos fundadores. No ano de 1994, os sócios tomaram a importante decisão de focar no desenvolvimento de sistemas para esses dois segmentos.

No final da década de 2000, a empresa experimentou grande crescimento no número de funcionários e passou a sistematizar práticas de recursos humanos $(\mathrm{RH})$ a fim de gerenciar quase o dobro de pessoas, organizar as funções e as relações de trabalho. Nos anos seguintes, em decorrência dessa explosão de crescimento, a administração que era dividida pelos codirigentes em duas áreas, direção em pequenas empresas. Revista de Empreendedorismo e Gestão de Pequenas Empresas, v.5, n.3, 2016. 
técnica e comercial, passou a ser apoiada por departamentos específicos, criados para facilitar a gestão.

Em 2014, ao perceberem a proximidade de mudança do patamar de PE para média empresa, os dois codirigentes atuantes decidiram estruturar o futuro por meio da contratação de consultoria especializada. A expectativa foi de consolidar o crescimento da empresa com a prestação de serviços de qualidade.

A PE Delta, localizada na cidade de São Paulo é a mais nova dentre as quatro estudadas. Foi fundada no ano de 2010, contudo, anteriormente sua equipe de direção já havia dado os primeiros passos no trabalho conjunto para criar um produto, o qual levaria à formalização dessa equipe e à fundação da Delta. A equipe de direção é também a mais jovem, já que dos três codirigentes, o mais velho encontra-se com 35 anos. A empresa desenvolve softwares e também administra projetos de sistemas. Atualmente são vinte e seis pessoas atuantes no desenvolvimento de projetos, vinte e três funcionários e os três codirigentes que também realizam as consultorias.

Ainda em situação informal, essa equipe criou um produto denominado Delta, a partir da constatação coletiva de insatisfação com os respectivos empregos. Dentre os codirigentes, dois eram sócios minoritários com uma pequena porcentagem na sociedade da empresa na qual trabalharam por mais de 10 anos. Nela ingressaram quando ainda era uma PE, contudo, como cresceu e tornou-se demasiadamente burocratizada, os codirigentes sócios passaram a não concordar com a política de gestão instituída.

Dessa insatisfação e a partir de conversas informais, ocorreu a associação entre os codirigentes que, além de colegas de trabalho, eram dois grandes amigos. O terceiro codirigente também partilhava as discordâncias e foi incluído logo em seguida na equipe de direção informal. Diante disso, decidiram tornarem-se donos do próprio negócio. Deixaram seus empregos e formalizaram a empresa. A porcentagem na sociedade ficou assim distribuída: codirigentes um e dois com $40 \%$ e o três, mais novo e menos experiente, com 20\%. Dentro de três meses, eles conseguiram lançar o produto Delta, empenharam-se em vendê-lo, mas como não obtiveram sucesso, buscaram alternativas de sobrevivência para o negócio. 
Nessa primeira crise, identificaram os serviços de consultoria em sistema como possível solução, mas também perceberam as dificuldades de trabalhar nessa área. Vencidas as dificuldades de atuação em serviços, identificaram também a oportunidade de abrir a equipe de direção e incluir mais um membro que contribuísse justamente com esse requisito.

\section{Processos de Decisão Estratégica (PDE) e Aprendizagem em Circuito Duplo}

Um resumo dos principais PDEs feitos pelos codirigentes em cada PE da amostra nos últimos cinco anos é apresentado no Quadro 1. Esses PDEs foram a base empírica considerada para a realização da pesquisa. Estão dispostos em ordem cronológica em cada linha.

QUADRO 1 - PROCESSOS DE DECISÃO ESTRATÉGICA (PDE) DAS QUATRO PES ESTUDADAS.

\begin{tabular}{|l|l|l|}
\hline PE & \multicolumn{3}{|c|}{ PDE identificados } \\
\hline Alpha & $\begin{array}{l}\text { Especialização em Softwares (2009); Formação da equipe de direção (2010); } \\
\text { Profissionalização dos serviços (2011); Reformulação da precificação (2013); } \\
\text { Reestruturação do sistema (2014). }\end{array}$ \\
\hline Beta & $\begin{array}{l}\text { Contratação de Consultoria Internacional (2009); Estabelecimento de Parceria (2011); } \\
\text { Controle de qualidade e especificações (2012); Sociedade com os parceiros (2014). }\end{array}$ \\
\hline Gama & $\begin{array}{l}\text { Inserção de práticas de gestão de Recursos Humanos (RH) (2009); Aperfeiçoamento do } \\
\text { software para CEASA (2010) Departamentalização de setores específicos (2012); Nova } \\
\text { identidade visual da empresa (2013); Contratação de consultoria especializada (2014). }\end{array}$ \\
\hline Delta & $\begin{array}{l}\text { Criação do produto Delta (2010); De produto para consultorias (2011); Exploração de } \\
\text { diferencial no design de softwares (2012); Entrada e saída de sócio na equipe de direção } \\
\text { (2012-2014). }\end{array}$ \\
\hline
\end{tabular}

FONTE: Os autores (2016).

Pode-se dizer que os codirigentes das PEs de base tecnológica estudadas são dinâmicos, quando se trata de processos de decisão estratégica. Nos quatro casos, os entrevistados relataram importantes decisões dos últimos cinco anos. Notou-se que a quantidade de decisões tomadas é notável do ponto de vista da "sabedoria convencional", termo utilizado por Gibcus e Van Hoesel (2004, p. 8), ao se referirem à concepção comum de que PEs quase não apresentam atividade econômica dinâmica.

TAVARES, T. S.; LIMA, E. Aprendizagem e decisão estratégica realizadas por equipes de direção em pequenas empresas. Revista de Empreendedorismo e Gestão de Pequenas Empresas, v.5, n.3, 2016. 
Muitas pesquisas têm adotado como foco central a investigação em torno da dicotomia: presença ou ausência de gestão estratégica nas PEs. Pode-se constatar nas PEs estudadas que há esse tipo de gestão, sobretudo nos processos de decisão estratégica identificados, todavia, notou-se que ocorre de maneira diferente das grandes organizações.

Nelas, notou-se que os processos de decisão estratégica, diferentemente das grandes organizações, envolvem também situações mais pontuais de melhoria contínua. Embora pareça terem envolvido processos simples, tais decisões tiveram impacto importante na sobrevivência das PEs. Os relatos a seguir atestam essa constatação: "Na real, a gente toma decisões pequenas, o interessante é que, às vezes, até muito grande no impacto que vai ter pra empresa [...]" (codirigente três da Delta).

[...] Nós não temos decisões grandes aqui, a gente tem muitos marcos, mas são marcos que acontecem naturalmente...na minha opinião a decisão grande foi tomada em 1994, quando a gente deixou alguns setores e focou nesses setores que a gente trabalha até hoje (hotel e CEASA), mas a gente tem muito trabalho de melhoria continua e decisões super importantes nesse sentido [...] (codirigente dois da Gama).

Nas citações, os codirigentes da Gama e da Delta destacaram algo que se repetiu em todos os casos de PE estudados nesta pesquisa: processos de decisão estratégica relativamente simples, contudo, fundamentais para a continuidade dos negócios. Sendo assim, pode-se afirmar que os processos de decisão estratégica ocorrem nas PEs estudadas de modo diferente em relação ao verificado na literatura que estuda tomada de decisão nas grandes empresas.

De modo geral, entende-se que os processos estudados são caracterizados por: uma abordagem mais informal para o planejamento, o aprender fazendo, a participação direta dos codirigentes, suas preferências e características pessoais, como identificaram os estudos de Escribá-Esteve, Sanchez-Peinado e SanchezPeinado (2009), Liberman-Yaconi, Hooper e Hutchings (2010) e Alcantar e Ngwenyama (2015).

Além disso, identificou-se que a aprendizagem permeia todos os PDEs estudados. Para cada PDE do Quadro 1, foi possível identificar, via análise das interações dos codirigentes, a ocorrência da aprendizagem em circuito duplo direção em pequenas empresas. Revista de Empreendedorismo e Gestão de Pequenas Empresas, v.5, n.3, 2016. 
(ARGYRIS; SCHÖN, 1978), bem como os fatores de destaque dessa aprendizagem. O Quadro 2 lista tais fatores.

QUADRO 2 - FATORES DE DESTAQUE DA APRENDIZAGEM NAS PDES

\begin{tabular}{|c|l|}
\hline PE & \multicolumn{1}{|c|}{ Fatores de destaque } \\
\hline Alpha & $\begin{array}{l}\text { Mudança de pensamento; Questionamento das ações atuais; Mudança de } \\
\text { comportamento em relação ao negócio; Redefinição de objetivos. }\end{array}$ \\
\hline Beta & $\begin{array}{l}\text { Reconhecimento de fraquezas; Mudança de pensamento; Interação emocional; } \\
\text { Mudança de comportamento em relação ao negócio. }\end{array}$ \\
\hline Gama & $\begin{array}{l}\text { Correção de erros; Questionamento das ações atuais; Reconhecimento de fraquezas; } \\
\text { Mudança de comportamento em relação ao negócio. }\end{array}$ \\
\hline Delta & $\begin{array}{l}\text { Redefinição de objetivos; Mudança de comportamento em relação ao negócio; } \\
\text { Mudança de pensamento; Questionamento dos pressupostos vigentes. }\end{array}$ \\
\hline
\end{tabular}

FONTE: Os autores (2016).

Os fatores do Quadro 2 são os mais frequentes nos PDEs apresentados no Quadro 1. Esses fatores constaram da análise dentre os códigos (GLASER; STRAUSS, 1967) mais importantes no tratamento dos dados feito com o software Atlas-ti, com o qual foram ligados a trechos dos dados, mais frequentemente de entrevistas. Tais códigos foram estabelecidos a partir do referencial teórico previamente construído.

Alguns códigos acabaram por ser pouco empregados e em apenas um caso de PE, enquanto outros se repetem nos diferentes casos. Por esse motivo, se construiu a Figura 1 na análise intracaso, figura representativa dos fatores de destaque essenciais a todas as PE estudadas. Na figura, também se encontra a síntese dos desdobramentos da aprendizagem em circuito duplo ligados aos PDEs identificados. 
FIGURA 1 - FATORES DE DESTAQUE ESSENCIAIS DAS PES

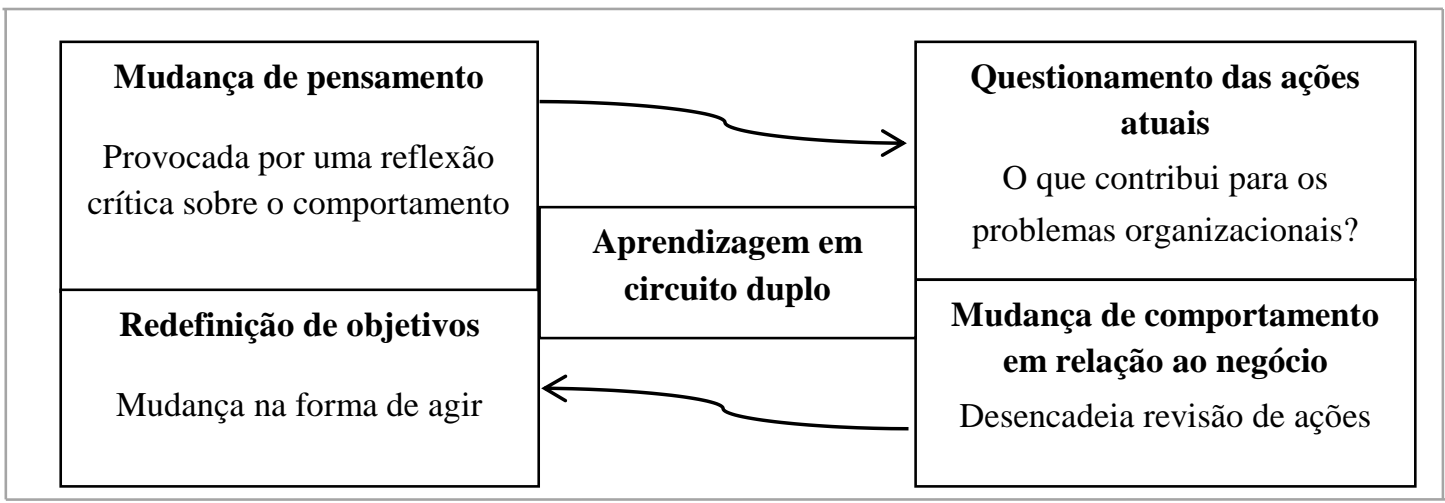

FONTE: Os autores (2016).

Nota-se que quatro fatores tiveram maior destaque: mudança de comportamento em relação ao negócio, questionamento das ações atuais, redefinição de objetivos e mudança de pensamento. Esses fatores repetiram-se na análise dos quatro casos de $\mathrm{PE}$.

A aprendizagem em circuito duplo ocorreu nos PDEs e desencadeou mudanças de pensamento e de ação estratégica dos codirigentes. A seguir, no Quadro 3, os produtos dessa aprendizagem ocorrida nos PDEs serão explicitados para cada processo e no caso de cada PE. 
QUADRO 3 - APRENDIZAGEM NOS PDES

\begin{tabular}{|c|l|}
\hline PE & \multicolumn{1}{|c|}{ Produtos da aprendizagem em circuito duplo ocorrida nos PDEs } \\
\hline Alpha & $\begin{array}{l}\text { Foco na prestação de serviços de software; descentralizar da gestão; transformação } \\
\text { no modo de se relacionar com os clientes; reorganização dos processos internos; } \\
\text { atenção às práticas de mercado com a compreensão de erros na forma de se } \\
\text { trabalhar; crescer deixou de ser importante, prevaleceu a correção dos rumos da } \\
\text { empresa e a redefinição dos objetivos para crescer de forma estruturada; formação de } \\
\text { uma equipe de direção. }\end{array}$ \\
\hline Beta & $\begin{array}{l}\text { Entendimento de que, para a continuidade do desenvolvimento da empresa, seriam } \\
\text { necessárias alianças e parcerias; reconhecimento do mercado além dos laboratórios } \\
\text { da universidade e dos projetos de pesquisa financiados pelo governo; compreensão } \\
\text { das dificuldades de se conduzir uma empresa de pesquisa e desenvolvimento (P\&D) } \\
\text { traduzida na aplicação de estratégias de controle de qualidade; evolução da } \\
\text { mentalidade de empresa de P\&D para empresa de mercado. }\end{array}$ \\
\hline Gama & $\begin{array}{l}\text { Entendimento de que a expansão da empresa deveria vir acompanhada de } \\
\text { estruturação das diferentes relações com os funcionários; reconhecimento de mercado } \\
\text { promissor para o software CEASA; entendimento de que a expansão da empresa } \\
\text { deveria vir acompanhada de estruturação dos seus principais setores; percepção da } \\
\text { necessidade de modernizar via atualização da imagem dos produtos; evolução da } \\
\text { mentalidade de PE para média empresa. }\end{array}$ \\
\hline Delta & $\begin{array}{l}\text { Reconhecimento do fracasso do produto e da alternativa de prestação de serviços em } \\
\text { consultorias de software; compreensão da necessidade de explorar os serviços } \\
\text { prestados, agregando mais valor por meio de diferenciais explícitos nos softwares } \\
\text { construídos; percepção de que a expansão da empresa precisaria se apoiar no } \\
\text { aprofundamento de conceitos e teorias; frustração dessa perspectiva diante das fortes } \\
\text { divergências de pensamento na nova equipe de direção. }\end{array}$ \\
\hline
\end{tabular}

FONTE: Os autores (2016).

Tendo em vista que a pesquisa realizada constatou expressões de aprendizagem em circuito duplo nos processos de decisão estratégica realizados pelos codirigentes, os parágrafos seguintes destacarão algumas influências dessa aprendizagem.

A partir dos processos de detecção e correção de possíveis erros de conduta e do aproveitamento deles para incentivar o crescimento, os codirigentes conseguiram perceber e intervir no sistema de funcionamento de seus negócios de uma forma dinâmica e adaptativa.

Constatou-se que essa interação se tornou natural e, espontaneamente, despertou processos de aprendizagem em circuito duplo, os quais foram ocorrendo a partir do reconhecimento dos codirigentes de que o desenvolvimento da empresa dependia de uma revisão nos conceitos e nas práticas, embora no passado eles tenham permitido avanços.

TAVARES, T. S.; LIMA, E. Aprendizagem e decisão estratégica realizadas por equipes de direção em pequenas empresas. Revista de Empreendedorismo e Gestão de Pequenas Empresas, v.5, n.3, 2016. 
Todas as PEs de base tecnológica estudadas atuam em ambientes com alto nível de incerteza como, por exemplo, no caso da Beta que é ainda muito complexo. Logo, processos de decisão estratégica puramente racionais não são adequados, pois, para serem eficazes, precisam lançar mão também de formas intuitivas em reflexões sobre os processos de decisão estratégica (LUBATKIN et al., 2006; VERREYNNE; MEYER; LIESCH, 2014).

Pode-se dizer que a aprendizagem apoiou o pensamento intuitivo nesses processos e conferiu-lhes alguma lógica, pois houve o questionamento e a revisão de pressupostos, ou seja, uma forma de pensamento não analítico racional, mas de avaliação dos comportamentos em relação ao negócio, que, de modo diferente, trouxe informações e permitiu sua interpretação nos processos de decisão estratégica.

A aprendizagem em circuito duplo significou uma reflexão crítica nessas PEs. Isso fez com que os codirigentes realizassem os processos de decisão estratégica com vistas a reverem seus comportamentos. Ela desencadeou mudanças profundas de pensamento e, consequentemente, de ações, conforme explicam Argyris e Schön (1978). Envolveu mudança de padrões vigentes por meio da interrogação de valores e revisão de conceitos, houve questionamentos de valores que alimentavam os processos de decisão nas PEs e, por fim, resultaram em ações, ou seja, entendimento do que se precisava mudar em conexão com as ações de mudança (ARGYRIS; SCHÖN, 1978).

Esse processo complexo registra o movimento da aprendizagem em circuito duplo descrito por Argyris e Schön (1978) e ocorrido nas PEs. Complexo porque foi capaz de mudar a mentalidade dos codirigentes nos processos de decisão estratégica, assim como deslocar suas crenças mais arraigadas.

Nos processos de decisão estratégica, evidenciou-se que os codirigentes utilizaram muito mais a aprendizagem em circuito duplo como combustível para deliberá-los do que procedimentos racionais analíticos e formais. Assim, interpretouse que a maneira de aprender e desenvolver os processos de decisão dessas PEs foi principalmente por meio da aprendizagem coletiva, como sugerido nos estudos de Wyer e Mason (1998), Bresman e Zellmer-Bruhn (2013), Yoon, Kim e Song (2015) e Wolff, Pett e Ring (2015).

TAVARES, T. S.; LIMA, E. Aprendizagem e decisão estratégica realizadas por equipes de direção em pequenas empresas. Revista de Empreendedorismo e Gestão de Pequenas Empresas, v.5, n.3, 2016. 
Como consequência, esse tipo de aprendizagem ocorrido nas PEs dependeu muito da capacidade e da vontade dos codirigentes de mudarem a mentalidade existente a respeito de como conduzir seus negócios. As principais partes interessadas, com as quais um dirigente interage, também podem contribuir e desencadeiam ajustes no seu modelo mental. Especialmente se há o desafio de concepções em uma dada situação, como ocorre nas equipes de direção pesquisadas. Nelas, os codirigentes inclusive oferecem, em suas interações, os benefícios da troca de experiências.

Nesse sentido, os resultados deste estudo também demonstram que aprender é um processo de adaptação e corroboram a pesquisa de Chaston, Badger, Sadler-Smith (2001), os quais enfatizaram que os dirigentes estudados continuavam com determinado curso de ação se percebessem a possibilidade de resultados positivos. Entretanto, mudavam suas ações se associassem os resultados a aspectos negativos, o que demonstra esse processo de aprendizagem, especialmente diante de eventos críticos. Semelhantemente, notou-se a forma com que os codirigentes das PEs estudadas aprenderam em seus diferentes processos de decisão estratégica e ganharam compreensão renovada sobre as estratégias organizacionais.

\section{Considerações Finais}

Este estudo buscou compreender como a aprendizagem em circuito duplo contribui para o processo de decisão estratégica de equipes de direção em PE. Para tanto, analisaram-se dados relativos a processos de decisão estratégica (PDE) que resultaram em mudanças importantes nos rumos das PEs estudadas. $O$ estudo converge com outras pesquisas de cunho descritivo quanto à preponderância da aprendizagem em situações relativamente informais, e não do planejamento estratégico formal, na gestão estratégica das PEs. Todas as PEs de base tecnológica estudadas atuam em ambientes com alto nível de incerteza. Logo, processos de decisão estratégica puramente racionais, com base na previsibilidade, não são adequados para essas empresas.

TAVARES, T. S.; LIMA, E. Aprendizagem e decisão estratégica realizadas por equipes de direção em pequenas empresas. Revista de Empreendedorismo e Gestão de Pequenas Empresas, v.5, n.3, 2016. 
Pode-se afirmar que, nos últimos cinco anos, a capacidade de os codirigentes aprenderem coletivamente em circuito duplo proporcionou às PEs estudadas uma necessária capacidade de resposta às oportunidades e ameaças que enfrentaram. Tais respostas apoiaram-se no pensamento intuitivo permeando os processos de decisão estratégica e auxiliando no questionamento e na revisão de pressupostos, assim como na geração de conhecimentos. Isso faz com que se reforce a noção já sugerida pela literatura de que a aprendizagem dos dirigentes é central na gestão estratégica das PEs (WYER; MASON, 1998).

A aprendizagem em circuito duplo significou nessas PEs uma reflexão crítica. Pode-se dizer que ela desencadeou mudanças profundas de pensamento e, consequentemente, de ação, conforme explicam Argyris e Schön (1978). Envolveu mudança de padrões vigentes por meio da interrogação de valores e revisão de conceitos, houve questionamentos de valores que alimentavam os processos de decisão estratégica nas PEs e, por fim, resultaram em novas ações promotoras de mudança, a partir do entendimento de que (e do que) se precisava mudar (ARGYRIS; SCHÖN, 1978).

Alguns padrões evidenciados na análise dos PDEs permitiram caracterizar o fenômeno estudado nas quatro PEs da amostra. Notou-se que nelas não há rotinas de realização das decisões estratégicas, as quais, talvez, pudessem simplificar o processo de decisão. Notou-se também que os PDEs são muito influenciados por preferências individuais dos codirigentes, principalmente nas empresas familiares.

As PEs tecnológicas enfrentaram alterações frequentes de seu contexto, muitas delas inconsistentes com padrões de pensamento e ação do passado. Por isso, regulamentação governamental (controle de qualidade nos laboratórios para a Beta), desenvolvimento e aplicação de novas tecnologias (software na nuvem para a Alpha), novos concorrentes internacionais (no setor de hotelaria para a Gama) e espaços no mercado (prestação de serviços em consultoria para a Delta), todos provocaram impactos significativos nas PEs estudadas.

Diante dessas alterações, destacou-se a relação de confiança entre os codirigentes, sobretudo quanto às habilidades uns dos outros, o que fez gerar processos participativos de decisão estratégica, nos quais não foi constatada a dominação por parte de qualquer um dos codirigentes. O equilíbrio da participação 
dos codirigentes nos PDEs foi um facilitador da aprendizagem em circuito duplo. Nas PEs Alpha e Beta, por exemplo, a contribuição técnica e aquela pautada em experiências passadas de cada codirigente se complementaram na maior parte dos PDEs identificados, evidenciando a complementaridade e o trabalho conjunto.

O modo de ocorrência das PEs estudadas é bem diferente de modelos prontos inspirados no planejamento estratégico e normalmente encontrados na literatura. A diferença mais importante é que, longe de serem racionais-formais, sofisticados e de complexa estrutura, mostraram-se bastante simples. Os métodos informais de decisão predominam. Alcantar e Ngwenyama (2015) também encontraram resultados semelhantes ao estudarem dirigentes de PE, que tomam decisões estratégicas predominantemente com base em suas experiências.

A partir deste estudo, fazem-se algumas sugestões para pesquisas futuras a serem analisadas na realidade das PEs. As de serviços encontram-se subrepresentadas na pesquisa em estratégia. Assim, há uma necessidade de mais pesquisas nessa área, posto que o setor de serviços vem crescendo e se tornando economicamente representativo, conforme defendem Liberman-Yaconi, Hooper e Hutchings (2010).

Investigações que contemplem comparações entre grandes empresas e PEs são bem-vindas também. Nelas, a interação e aprendizagem dos codirigentes em PDE poderia ser o foco, permitindo confrontar e destacar os elementos diferentes que contribuem especificamente nesse processo nas PEs. Esta pesquisa demonstrou características diferentes das citadas na literatura, revelando a realidade singular das PEs.

A sugestão de potenciais estudos, que inclusive ajudariam na superação das limitações deste trabalho, envolve métodos de coleta de dados diferentes dos adotados na pesquisa aqui apresentada. Considerando-se que no momento das entrevistas as recordações que levaram aos relatos sobre como as decisões foram tomadas nos últimos cinco anos podem ter sido muito mais elaboradas e racionalizadas do que de fato foi o ocorrido na época da tomada de decisão, sugerese como estudo futuro, pesquisas longitudinais. Esse tipo de pesquisa possibilitaria o acompanhamento dos processos de decisão, minimizando possíveis vieses que os relatos em retrospectiva possam causar.

TAVARES, T. S.; LIMA, E. Aprendizagem e decisão estratégica realizadas por equipes de direção em pequenas empresas. Revista de Empreendedorismo e Gestão de Pequenas Empresas, v.5, n.3, 2016. 
Além disso, em termos de condução da pesquisa, sugere-se o uso do método etnográfico. Dada a possibilidade de aprofundamento concedida por ele, acredita-se que poderia contribuir muito mais no detalhamento sobre os elementos que influem em como os codirigentes realizam seus processos de decisão estratégica.

Espera-se que os resultados desta pesquisa possam colaborar para melhorias nos processos de decisão estratégica na PE e para o preenchimento de lacunas existentes nessa área de conhecimento. Sobretudo, considerando o multifacetado e específico contexto da PE, como alertam Verreynne, Meyer e Liesch (2014).

\section{Referências:}

ALCANTAR, J.; NGWENYAMA, O. Top management capabilities for SME's market entry decisions. In: INTERNATIONAL ASSOCIATION FOR MANAGEMENT OF TECHNOLOGY - IAMOT, 2015. Conference Proceedings... 2015, p. 1348-1362. Disponível em: <http://iamot2015.com/2015proceedings/documents/P179.pdf>. Acesso em: 10 abr. 2016.

ALEXANDERSSON, A.; FRIDOLF, A. The Process of Distributor Selection among SMEs. A multiple-case study in the Healthcare Sector. Master Degree Project in International Business and Trade, 2015.

ARGYRIS, C. Double-loop learning, teaching, and research. Academy of Management Learning and Education, v. 1, n. 2, p. 206-218, 2002.

ARGYRIS, C. Single-loop and double-loop models in research on decision making. Administrative Science Quarterly, v. 21, n. 3, p. 363-375, 1976.

ARGYRIS, C.; SCHÖN, D. A. Organizational Learning: a Theory of Action Perspective. Reading: Addison-Wesley Series on Organization Development, 1978.

BRESMAN, H.; ZELLMER-BRUHN, M. The structural context of team learning: effects of organizational and team structure on internal and external learning. Organization Science, v. 24, n. 4, p. 1120-1139, 2013.

BROUTHERS, K. D.; ANDRIESSEN, F.; NICOLAES, I. Driving blind: Strategic decision making in small companies. Long Range Planning, v. 31, n. 1, p. 130-138, 1998.

TAVARES, T. S.; LIMA, E. Aprendizagem e decisão estratégica realizadas por equipes de direção em pequenas empresas. Revista de Empreendedorismo e Gestão de Pequenas Empresas, v.5, n.3, 2016. 
BRUNNINGE, O.; NORDQVIST, M.; WIKLUND, J. Corporate governance and strategic change in SMEs: the effects of ownership, board composition and top management teams. Small Business Economics, v. 29, n. 2, p. 295-308, 2007.

CARMELI, A. Top Management Team Behavioral Integration and the Performance of Service Organizations. Group \& Organization Management, v. 3, n. 6, p. 712-735, 2008.

CARMELI, A.; TISHLER, A.; EDMONDSON, A. C. CEO relational leadership and strategic decision quality in top management teams: The role of team trust and learning from failure. Strategic Organization, v. 10, n. 1, p. 31-54, 2012.

CHASTON, I.; BADGER, B.; SADLER-SMITH, E. Organizational learning: an empirical assessment of process in small UK manufacturing firms. Journal of Small Business Management, v. 39, n. 2, p. 139-151, 2001.

COOPER, A. C. Strategic management: new ventures and small firms. Long Range Planning, v.14, n. 5, p. 39-45, 1981.

CRESWELL, J. W. Projeto de pesquisa: Métodos qualitativo, quantitativo e misto. Porto Alegre: Bookman e Artmed., 2007.

EASTERBY-SMITH, M.; ANTONACOPOULOU, E.; SIMM, D.; LYLES, M. Constructing Contributions to Organizational Learning Argyris and the Next Generation. Management Learning, v. 35, n. 4, p. 371-380, 2004.

EISENHARDT, K. M. Building Theories from Case Study Research. Academy of Management Review, v.14, n. 4, p. 522-550, 1989.

EISENHARDT, K. M.; ZBARACKI, M. J. Strategic decision making. Strategic Management Journal, v. 13, n. 2, p. 17-37, 1992.

EISENHARDT, K. M.; KAHWAJY, J. L.; BOURGEOIS III, L. J. How management teams can have a good fight. Harvard Business Review, v. 75, n. 4, p. 77-90, 1997.

ESCRIBÁ-ESTEVE, A.; SANCHEZ-PEINADO, L.; SANCHEZ-PEINADO, E. The influence of Top management teams in the strategic orientation and performance of small and medium-sized enterprises. British Journal of Management, v. 20, n. 3, p. 581-597, 2009.

FAGUNDES, F. M.; GIMENEZ, F. A. P. Ambiente, estratégia e desempenho em micro e pequenas empresas. REBRAE - Revista Brasileira de Estratégia, v. 2. n. 2, p. 133-146, 2009.

FINKELSTEIN, S.; HAMBRICK, D. Strategic leadership: Top executives and their effects on organizations. Minneapolis: West Pub. Co., 1996.

TAVARES, T. S.; LIMA, E. Aprendizagem e decisão estratégica realizadas por equipes de direção em pequenas empresas. Revista de Empreendedorismo e Gestão de Pequenas Empresas, v.5, n.3, 2016. 
FULLER-LOVE, N. Management development in small firms. International Journal of Management Reviews, v. 8, n. 3, p.175-190, 2006.

GELDENHUYS, D. J.; CILLIERS, F. Transforming a small business: A learning intervention. SA Journal of Industrial Psychology, v. 38, n. 2, p. 147-155, 2012.

GIBCUS, P.; VAN HOESEL, P. H. M. Transforming an Idea into a Strategic Decision in SMEs: The Underlying Decision-Making Process. EIM Business \& Policy Research, 2004.

GLASER, B.; STRAUSS, A. The Discovery of Grounded Theory: Strategies for Qualitative Research. London: Wiedenfeld and Nicholson, 1967.

GODOI, C. K.; MATTOS, P. L. de. Entrevista qualitativa: instrumento de pesquisa e evento dialógico. In: GODOI, C. K.; BANDEIRA-DE-MELLO, R.; SILVA, A. B. (Org.). Pesquisa Qualitativa em Estudos Organizacionais: paradigmas, estratégias e métodos. São Paulo: Editora Saraiva, 2006.

GREENE, P. G.; BRUSH, C. G.; BROWN, T. E. Resources in small firms: an exploratory study. Journal of Small Business Strategy, v. 8, n. 2, p. 25-40, 2015.

HAMBRICK, D. Fragmentation and the other problems CEOs have with their top management teams. California Management Review, v. 37, n. 1, p. 110-127, 1995.

HAMBRICK, D.; MASON, P. Upper echelons: the organization as a reflection of its top managers. Academy of Management Review, v. 9, n. 2, p. 193-206, 1984.

IBRAHIM, A. B. Strategy Types and Small Firms' Performance An Empirical Investigation. Journal of Small Business Strategy, v. 4, n. 1, p. 13-22, 2015.

IBRAHIM, A. B.; DUMAS, C.; MCGUIRE, J. Strategic decision making in small family firms: an empirical investigation. Journal of Small Business Strategy, v. 12, n. 1, p. 80-90, 2015.

KANTAMARA, P.; VATHANOPHAS, V. Single-Loop vs. Double-Loop Learning: An Obstacle or a Success Factor for Organizational Learning. International Journal of Education and Research, v. 2, n. 7, p. 55-62, 2014.

LECHLER, T. Social Interaction: A Determinant of Entrepreneurial Team Venture Success. Small Business Economics, v. 16, n. 1, p. 263-278, 2001.

LIBERMAN-YACONI, L.; HOOPER, T.; HUTCHINGS, K. Toward a model of understanding strategic decision-making in micro-firms: exploring the Australian information technology sector. Journal of Small Business Management, v. 48, n. 1, p. $70-95,2010$.

LIMA, E. Estratégia de Pequenas e Médias Empresas: Uma Revisão. REGE Revista de Gestão USP, v. 17, n. 2, p. 169-187, 2010.

TAVARES, T. S.; LIMA, E. Aprendizagem e decisão estratégica realizadas por equipes de direção em pequenas empresas. Revista de Empreendedorismo e Gestão de Pequenas Empresas, v.5, n.3, 2016. 
LIMA, E. Les liens entre représentations entrepreneuriales et vision partagée. Revue Internationale de Psychosociologie, v. 18, n. 1, p. 199-225, 2012.

LIU, F.; MAITLIS, S. Emotional dynamics and strategizing processes: a study of strategic conversations in top team meetings. Journal of Management Studies, v. 51, n. 2, p. 202-234, 2014

LOHRKE, F. T.; FRANKLIN, G. M.; KOTHARI, V. B. Top Management Team Heterogeneity and SME Export Performance: Investigating the Role of Environmental Uncertainty. Journal of Small Business Strategy, v. 14, n. 1, p. 86-102, 2015.

LUBATKIN, M. H.; SIMSEK, Z.; LING, Y.; VEIGA, J. F. Ambidexterity and performance in small-to medium-sized firms: The pivotal role of top management team behavioral integration. Journal of management, v. 32, n. 5, p. 646-672, 2006.

MAZZAROL, T. W.; REBOUD, S. The strategic management process. IN: MAZZAROL, T. W.; REBOUD, S. (Org.). The Strategy of Small Firms: strategic management and innovation in the small firm. Northampton USA: Edward Elgar Publishing, 2009.

MCCARTHY, B.; LEAVY, B. Phases in the Strategy Formation Process: An Exploratory Study of Irish SMEs. IBAR, v. 21, n. 2, p. 55-80, 2000.

MILES, M. B.; HUBERMAN, A. M. Qualitative data analysis: an expanded sourcebook. California: Sage, 1994.

MORGAN, G.; SMIRCICH, L. The case for qualitative research. Academy of Management Review, v. 5, n. 4, p. 491-500, 1980.

MUELLER, C. B.; NAFFZIGER, D. W. Strategic planning in small firms: Activity and process realities. Journal of Small Business Strategy, v. 10, n. 1, p. 78-85, 2015.

OLSON, B. J.; PARAYITAM, S.; BAO, Y. Strategic decision making: The effects of cognitive diversity, conflict, and trust on decision outcomes. Journal of Management, v. 33, n. 2, p. 196-222, 2007.

PANDIT, N. R. The Creation of Theory: a Recent Application of the Grounded Theory Method. The Qualitative Report, v. 2, n. 4, p. 378-299, 1996.

PECH-VARGUEZ, J. L.; CISNEROS, L.; GENIN, E.; CORDOVA, H. Cohérence et cohésion de l'équipe de direction dans la PME. Revue internationale P.M.E., v. 23, n. 3, p. $188-209,2010$.

PETTIGREW, A. M. The Character and Significance of Strategy Process Research. Strategic Management Journal, v. 13, n. 1, p. 5-16, 1992.

TAVARES, T. S.; LIMA, E. Aprendizagem e decisão estratégica realizadas por equipes de direção em pequenas empresas. Revista de Empreendedorismo e Gestão de Pequenas Empresas, v.5, n.3, 2016. 
REUBER, R.; FISCHER, E. The influence of the management team's international experience on the internationalization behaviors of SMEs. Journal of International Business, v. 2, n. 8, p. 807-825, 1997.

RUEF, M.; ALDRICH, H. E.; CARTER, N. M. The structure of founding teams: homophily, strong ties, and isolation among U.S. entrepreneurs. American Sociological Review, v. 68, n. 2, p. 195-222, 2003.

SAPIE, N. M. et al. Organization Learning Determinants of Innovative Work Behavior: Study of Malaysian Small and Medium Enterprises at Three Cities Selected In Malaysia. Journal for Studies in Management and Planning, v. 1, n. 3, p. 549-560, 2015.

SEBRAE, SERVIÇO BRASILEIRO DE APOIO ÀS MICRO E PEQUENAS EMPRESAS. Anuário do trabalho na micro e pequena empresa: 2010-2011. 4. ed., São Paulo: Dieese, 2011.

SMITH, T.; BARRETT, R. Informal e-learning: the case of small business owners. Education + Training, v. 56, n. 2/3, p. 219-232, 2014

STRAUSS, A.; CORBIN, J. Basics of qualitative research: techniques and procedures for developing Grounded Theory. 3 ed. Thousand Oaks: Sage, 2008.

TIHULA, S.; HUOVINEN, H. Reasons and Situational Factors behind the Formation of Management Teams and Other Teams in Small Firms. In: FINK, M.; KRAUS, S. The Management of Small and Medium Enterprises. New York: Routledge, 2009.

VERREYNNE, M.; MEYER, D.; LIESCH, P. Beyond the Formal-Informal Dichotomy of Small Firm Strategy-Making in Stable and Dynamic Environments. Journal of Small Business Management, v. 1, 2014.

WOLFF, J.; PETT, T. L.; RING, J. K. Small firm growth as a function of both learning orientation and entrepreneurial orientation: An empirical analysis. International Journal of Entrepreneurial Behavior \& Research, v. 21, n. 5, p. 709-730, 2015.

WU, C.; YOUNG, A. Critical operating problems and survival rates in small firms: A look at small business institute clients. Journal of Developmental Entrepreneurship, v. 7, n. 1, p. 1-23, 2002

WYER, P.; MASON, J. An organisational learning perspective to enhancing understanding of people management in small businesses. International Journal of Entrepreneurial Behaviour \& Research, v. 4, n. 2, p. 112-128, 1998.

WYER, P.; MASON, J.; THEODORAKOPOULOS, N. Small business development and the "learning organisation". International Journal of Entrepreneurial Behaviour \& Research, v. 6, n. 4, p. 239-256, 2000.

TAVARES, T. S.; LIMA, E. Aprendizagem e decisão estratégica realizadas por equipes de direção em pequenas empresas. Revista de Empreendedorismo e Gestão de Pequenas Empresas, v.5, n.3, 2016. 
YIN, R. K. Estudo de caso: planejamento e métodos. 4. ed. Porto Alegre: Bookman, 2010.

YOON, W.; KIM, S.; SONG, J. Top management team characteristics and organizational creativity. Review of Managerial Science, p. 1-23, 2015. 